\title{
Regulation of Life Extension Factor Klotho on Depressive-like Behaviors via Modulation of GluN2B Function in the Nucleus Accumbens
}

\section{Han-jun Wu}

Jiangxi Mental Hospital \& Affiliated Mental Hospital of Nanchang University

Liu-er Liu

Jiangxi Mental Hospital \& Affiliated Mental Hospital of Nanchang University

\section{Wen-ning Wu}

School of Basic Medical Sciences, Anhui Medical University

Jin-qiong Zhan

Jiangxi Mental Hospital \& Affiliated Mental Hospital of Nanchang University

Yi-heng Li

Jiangxi Mental Hospital \& Affiliated Mental Hospital of Nanchang University

Chun-nuan Chen

The Second Affiliated Hospital, Fujian Medical University

\section{Shu-zhen Jiang}

Jiangxi Mental Hospital \& Affiliated Mental Hospital of Nanchang University

Jian-wen Xiong

Jiangxi Mental Hospital \& Affiliated Mental Hospital of Nanchang University

Zhi-Min Yu

Jiangxi Mental Hospital \& Affiliated Mental Hospital of Nanchang University

\section{Wei Wang}

\section{Bo Wei}

Jiangxi Mental Hospital \& Affiliated Mental Hospital of Nanchang University

\section{Yuan-Jian Yang ( $\nabla$ yuanjimyang@yeah.net )}

Jiangxi Mental Hospital \& Affiliated Mental Hospital of Nanchang University https://orcid.org/00000002-0175-5646

\section{Article}

Keywords: Depression, Klotho, N-methyl-D-aspartate receptor (NMDAR), GluN2B, nucleus accumbens (NAc)

Posted Date: January 3rd, 2022 
DOI: https://doi.org/10.21203/rs.3.rs-1218831/v1

License: (c) (1) This work is licensed under a Creative Commons Attribution 4.0 International License. Read Full License 


\section{Abstract}

Klotho is a life extension factor that has an ability to regulate the function of GluN2B-containing Nmethyl-D-aspartate receptors (NMDARs), whose dysfunction in the nucleus accumbens (NAc) underlies critical aspects of the pathophysiology of major depression. Here we study the functional relevance of klotho in the pathogenesis of depression. A chronic social defeat stress paradigm, where mice are either categorized as susceptible or unsusceptible group based on their performance in a social interaction test, was used in this study. We found that the expression of klotho was largely decreased in the NAc of susceptible mice when compared to control or unsusceptible group. Genetic knockdown of klotho in the NAc induced depressive-like behaviors in naive mice, while overexpression of klotho produced an antidepressive effect in normal mice and ameliorated the depressive-like behaviors in susceptible mice. Molecularly, knockdown of klotho in the NAc resulted in selective decreases of total and synaptic GluN2B expression that were identical to susceptible mice. Elevation of klotho in the NAc reversed the reductions of GluN2B expressions, as well as altered synaptic transmission and spine density in the NAc of susceptible mice. Furthermore, blockade of GluN2B with a specific antagonist abolished the beneficial effects of klotho elevation in susceptible mice. Collectively, we demonstrated that klotho in the NAc modulates depressive-like behaviors by regulating the function of GluN2B-containing NMDARs. These results reveal a novel role for klotho in the pathogenesis of depression, opening new insights into the molecular basis of major depression.

\section{Introduction}

Depression is a common type of affective disorders that is characterized by marked and persistent low mood and loss of interest, leading to serious functional impairments in patients ${ }^{1}$. The Global Burden of Disease Study demonstrates that depression is the second leading cause of disability-adjusted life-years in $2020^{2}$. Numerous antidepressants are currently used in clinical practice, however, it still takes weeks for patients to get therapeutic effects and some patients are poor tolerant to antidepressants ${ }^{3}$.

Understanding of the pathophysiological mechanisms underlying major depression would shed light on the treatment of major depressive disorders.

The nucleus accumbens (NAc), a brain region located in the ventral aspect of the basal ganglia, is widely recognized as the center of reward and motivation ${ }^{4}$. NAc medium spiny neurons display a two-state membrane potential controlled by active channels and synaptic input ${ }^{5}$, thereby affecting the functional activity in networks that underlie cognition and behavior ${ }^{6}$. The NAc receives glutamatergic inputs from the medial prefrontal cortex (MPFC), basolateral amygdala and hippocampus. Accumulating evidence has shown that altered glutamatergic transmission in the NAc contributes importantly to the pathophysiology of depression ${ }^{7-10}$. Synaptic glutamate release in mPFC-NAc is decreased in mice exhibiting depressive-like behaviors ${ }^{9}$, and the decreased activity at glutamatergic synapses obstructs the later induction of long-term depression (LTD) in the NAc of these mice ${ }^{11,12}$. Our previous study revealed that chronic stress caused persistent downregulation of total and synaptic GluN2B, a N-methyl-D- 
aspartate receptor (NMDAR) subunit with key functions in learning and memory, in the NAc and disrupted the induction of NMDAR-dependent LTD of cortico-accumbal glutamatergic synapse, while restoration of GluN2B loss reversed stress-induced LTD deficit and alleviate depressive-like behaviors in chronic social defeat stress (CSDS)-susceptible mice, indicating that downregulation of GluN2B function in the NAc underlies the synaptic and behavioral adaptations to chronic stress ${ }^{8}$.

The klotho protein is a recently discovered protein that is associated with life extension. Overexpression of klotho extends life span, whereas loss of klotho leads to an accelerated aging and short life ${ }^{13-15}$. In addition to lifespan extension, it has also been linked to cognition and other neuropsychiatric disorders. For example, knockout of klotho resulted in memory retention deficits in mice ${ }^{16}$, while elevation of klotho expression can enhance hippocampus-dependent learning and memory in normal rodents and protect against cognitive decline in animal models of Alzheimer's disease $(A D)^{17-19}$. In a previous clinical observational study, AA Prather ${ }^{20}$ found that women under high chronic stress displayed significantly lower levels of serum klotho compared with low-stress controls. KLOTH gene variants influenced the response to selective serotonin reuptake inhibitors (SSRIs) in late-life major depressive disorder ${ }^{21}$. Electroconvulsive therapy (ECT), a highly effective antidepressant treatment, significantly enhanced the levels of klotho in the cerebrospinal fluid of geriatric patients with major depression ${ }^{22}$. However, whether klotho is involved in the pathogenesis of major depression remains unclear.

Klotho is highly expressed in the choroid plexus and neurons, as well as in the kidney and reproductive organs. Its transmembrane form can be released by sheddases and circulate in serum and cerebrospinal fluid throughout life ${ }^{23}$. There are studies demonstrating that klotho could enhance the function of GluN2B subunit ${ }^{18,19}$. Upregulation of klotho expression promoted hippocampal synaptic plasticity and cognition by enriching synaptic GluN2B in the hippocampus of mice, while blocking GluN2B abolished the beneficial effects of klotho elevation ${ }^{18}$. Elevating klotho expression in human amyloid precursor protein (hAPP) transgenic mice increased the abundance of GluN2B in the postsynaptic densities to improve spatial learning and memory ${ }^{24}$. In view of the important role of accumbal GluN2B loss in the pathophysiology of depression ${ }^{8}$, as well as the regulation of klotho on GluN2B function, it is possible that klotho in the NAc also fulfills important functions in the pathogenesis of major depression. To test this hypothesis, we first investigated whether the expression of klotho was changed in the NAc from mice displaying depressive-like behaviors and then explored the influences of modulating accumbal klotho expression on depressive-like behaviors in mice. By constructing stable adeno-associated virus vector system to regulate the expression of klotho in the NAc of mice, we demonstrated that klotho in the NAc modulated depressive-like behaviors in mice by regulating the surface stability of GluN2B subunit.

\section{Methods}

\section{Animals}


Adult male C57BL/6J mice (8-10 weeks old) and male CD1 mice (6-month-old) were purchased from Hunan SJA Laboratory Animal Co., Ltd (Changsha, Hunan, China). Mice were fed under standard conditions (12 h light/dark cycle; lights on from 07:00 to 19:00; $23 \pm 1^{\circ} \mathrm{C}$ ambient temperature; $55 \pm 10 \%$ relative humidity), with free access to food and water. All behavioral experiments were performed in the day and conducted in compliance with the Guide for the Care and Use of Laboratory Animals (8th edition, Institute of Laboratory Animal Resources on Life Sciences, National Research Council, National Academy of Sciences, Washington DC). This research was approved by the Review Committee for the Use of Human or Animal Subjects of Jiangxi Mental Hospital. Experimenters were blinded to experimental group and the order of testing was counterbalanced during behavioral experiments.

\section{Social Interaction Test (SIT)}

Social avoidance behavior was assessed with a novel CD1 mice in a two-stage social interaction test. In the first 3-min test (target absent), the defeated mice were allowed to freely explore an arena $(44 \times 44 \mathrm{~cm})$ containing a plexiglass and wire mesh enclosure $(10 \times 6 \mathrm{~cm})$ against one wall of the arena. In the second 3-min test (target present), the experimental mice were returned to the area with a novel CD1 mice enclosed in the plexiglass wire mesh cage. This allowed the animal to see, hear, and smell, but not physically contact. Time spent in the 'interaction zone' $(14 \times 26 \mathrm{~cm})$ surrounding the plexiglass wire mesh cage and 'distance travelled' within the arena was recorded by ANY-maze tracking software (ANY-maze, Wood Dale, IL). The segregation of susceptible and unsusceptible mice was based on the social interaction ratio, which was calculated as: time in interaction zone when target present/time in interaction zone when target absent $\times 100 \%$. Defeated mice with a social interaction ratio $<100 \%$ were defined as 'susceptible', while those with a social interaction ratio $\geq 100 \%$ were defined as 'unsusceptible'.

Additional experimental procedures and statistics are described in Supplement information.

\section{Results}

\section{CSDS Significantly Decreased Klotho Expression in the NAC of Susceptible Mice}

The CSDS has a good predictive validity for modeling the symptomatology of depression, thus we adopted this model to investigate the role of klotho in depression in this study. C57BL/ $6 \mathrm{~J}$ mice were exposed to 10 consecutive days of stress and then were designated as susceptible or unsusceptible mice based on social interaction ratios (Figure S1a). Compared to control and unsusceptible mice, susceptible mice displayed a significant decrease in the sucrose preference (Figure $\mathrm{S} 1 \mathrm{~b}, p<0.01$ ) and a significant increase in immobility time in both the FST and the TST (Figure S1c and d, $p<0.01$ ). These behavioral results demonstrated that CSDS could successfully induce depressive-like behaviors in mice. Therefore, CSDS susceptible mice were used as the model mice of major depression in the subsequent experiments.

To explore whether CSDS would result in change of klotho expression in the brain, the protein level of klotho was determined by western blot analysis. As shown in Figure 1a, klotho protein expression in the 
whole-brain was significantly decreased in susceptible mice compared with control or unsusceptible mice $(p<0.01)$. Then the expressions of klotho in brain regions closely related to depression, including PFC, hippocampus and NAc, were detected. As shown in Figure 1b, there was no significant difference in klotho expression in the PFC among control, unsusceptible and susceptible group. However, compared to control or unsusceptible mice, the levels of klotho in the hippocampus and NAc of susceptible mice were significantly decreased. Klotho proteins in the hippocampus and NAc of susceptible mice were reduced by $25.2 \pm 3.7 \%$ ( $p<0.05 \mathrm{vs}$. control) and $63.2 \pm 5.2 \%(p<0.01$ vs. control), respectively.

\section{Genetic Knockdown of Klotho in the NAc Induced Depressive-like Behaviors in Mice}

Given that the level of klotho protein was largely decreased in the NAc of mice after CSDS, we presumed that abnormal accumbal klotho signaling might contribute to the pathogenesis of depression. To test this hypothesis, we investigated whether downregulation of klotho expression in the NAc would result in depressive-like behaviors in mice (Figure 1c). We used an adeno-associated viral vector (AAV) to specifically reduce the expression of klotho in the NAc. AAV-klotho knockdown-GFP (KL-KD) or AAV-NullGFP (GFP) was stereotaxically infused into the NAc of naive mice. The expression of klotho in the NAc was detected 2 weeks after injection. We showed that numerous GFP-positive cells were found in the NAc region after injection (Figure 1d) and the protein expression of klotho in the NAc of KL-KD mice was significantly decreased when compared to control or GFP group (Figure $1 e, p<0.01$ vs. control), indicating that AAV-mediated knockdown of klotho in the NAc was successfully constructed.

Next, we examined whether mice with knockdown of klotho in the NAc displayed depressive-like behaviors. As expect, compared to control mice, the KL-KD mice had a significantly lower level of sucrose consumption (Figure 1f, $p<0.01 \mathrm{vs}$. control group). The immobility time of mice in the KL-KD group was also significantly increased in FST and TST (Figure $1 \mathrm{~g}$ and $\mathrm{h}, p<0.01 \mathrm{vs}$. control group). Open field test showed that there was no significant difference in traveled distance among groups $(p>0.05)$, while the time spent in the center square was significantly decreased in KL-KD mice (Figure $1 \mathrm{i}$ and $\mathrm{j}, p<0.01$ vs. control group), indicating that the behavioral alterations in KL-KD mice were not due to change in spontaneous locomotor activity, but rather depressive-like behaviors. AAV-control-GFP (GFP) mice did not display any behavioral difference from the control group. The results indicated that reduced nucleus accumbens klotho contributed to the pathogenesis of major depression.

\section{Genetic Overexpression of Klotho in the NAc Produces Antidepressive Effects in Both Normal and CSDS Susceptible Mice}

To verify the role of klotho signaling in depression, we investigated whether elevation of klotho expression in the NAc would affect depressive-like behaviors. We first tested the influence of elevating accumbal klotho on depressive-like behaviors in normal mice. AAV was employed to enhance klotho 
expression in the NAc. AAV-klotho overexpression-GFP (KL-OE) or AAV-Null-GFP (GFP) was stereotaxically infused into the NAc of normal mice. Two weeks after injection, the protein expression of klotho in the NAc of KL-OE mice was significantly increased when compared to control or GFP group (Figure S2a, $p<$ 0.01 vs. control or GFP group). Increased sucrose consumption in SPT and decreased immobility time in FST were observed in these KL-OE mice (Figure S2b and c, both $p<0.05 v s$. control group), indicating that elevation of accumbal klotho could exhibit an antidepressive effect in normal mice.

Then we further explored whether elevation of klotho expression in the NAc would attenuate the depressive-like behaviors in CSDS susceptible mice (Figure 2a). As shown in Figure 2b, KL-OE increased the protein expression of klotho in the NAc of susceptible mice to a level comparable to that of control group ( $p<0.01 v s$. susceptible or susceptible-GFP group). Results from social interaction test revealed that accumbal KL-OE significantly increased the exploration time in interaction zone and the interaction ratio in susceptible mice (Figure 2c, $p<0.01$ vs. susceptible or susceptible-GFP group). The decreased sucrose preference in susceptible mice was also significantly reversed by KL-OE (Figure 2d, $p<0.01$ vs. susceptible or susceptible-GFP group). Furthermore, accumbal KL-OE in susceptible mice shorten the immobility time in FST (Figure 2e) and TST (Figure 2f) to levels comparable to those of control group ( $p<$ $0.01 \mathrm{vs}$. susceptible or susceptible-GFP group). Open field test showed that accumbal KL-OE in susceptible mice did not affect the traveled distance of mice, but increased the time spent in the center (Figure 2g, $p<0.01 v s$. susceptible or susceptible-GFP group). These results demonstrated that elevation of klotho in the NAc ameliorated depressive-like behaviors of susceptible mice, confirming the critical modulatory effects of accumbal klotho on the pathogenesis of major depression.

\section{Knockdown of Klotho in the NAc Resulted in Selective Decreases of Total and Synaptic GluN2B that Were Identical to CSDS Susceptible Mice}

To explore the potential mechanisms underlying the modulatory effects of klotho on depressive-like behaviors, we turned our attention to NMDARs, whose dysfunction were demonstrated to mediate behavioral and synaptic adaptations to chronic stress ${ }^{8}$. We performed western blotting to examine the levels of NMDAR subunits and postsynaptic density protein 95 (PSD-95) in total protein homogenates of NAc 10 days after CSDS. As previous reported ${ }^{8}$, we showed that total protein expressions of GluN1 and GluN2A in the NAc were not changed after CSDS ( $p>0.05 \mathrm{vs}$. control), while the level of GluN2B subunit in the NAc of susceptible mice were significantly decreased compared to control group (Figure S3a, $p<$ 0.01). PSD-95 is a neuronal PDZ protein that associates with NMDARs at synapses to facilitate downstream intracellular signaling and modulate synaptic plasticity ${ }^{25}$ 26. Akin to GluN2B, PSD-95 displayed a significant decrease in the NAc of susceptible mice (Figure S3a, $p<0.01 v s$. control). Then we investigated whether downregulation of klotho in the NAc would result in similar changes in NMDAR subunit expression. As shown in Figure 3a, KL-KD in the NAc caused a specific decrease in total protein 
levels of GluN2B subunit and PSD-95, but did not alter total protein levels of GluN1 and GluN2A ( $p<0.01$ vs. control or GFP group).

The biological consequences of NMDAR activation mainly depend on whether the receptors are located in synaptic or extrasynaptic sites ${ }^{27}$. We therefore detected the surface expressions of NMDAR subunits in NAc of mice using a protein cross-linking assay that specifically detects synaptic proteins. Similar to previous report ${ }^{8}$, we showed that there was no difference in the levels of GluN1 and GluN2A in both surface pool and intracellular pool between control and susceptible mice (Figure S3b and c, $p>0.05$ ). However, a robust decrease in GluN2B was observed in the surface pool in susceptible mice (Figure S3d, $p<0.01)$. These results demonstrated a selective GluN2B downregulation in a specific subcellular compartment (surface membranes) in the NAc of depressive model mice. We next explored the influence of klotho downregulation on the expressions of NMDARs in the synapses of NAc. Similarly, there was no difference in the levels of GluN1 and GluN2A in both surface and intracellular pool between control and $\mathrm{KL}-\mathrm{KD}$ mice (Figure $3 \mathrm{~b}$ and $\mathrm{c}$ ), while a significant decrease was observed in GluN2B in the surface pool in $\mathrm{KL}-\mathrm{KD}$ mice (Figure $3 \mathrm{~d}, \mathrm{p}<0.01 \mathrm{vs}$. control or GFP group). These results demonstrated that knockdown of klotho produced an identical change in GluN2B expression to susceptible mice, suggesting that decrease of accumbal klotho resulted in depressive-like behaviors in mice via a selective downregulation of GluN2B at synapses of NAc.

\section{Genetic Overexpression of Klotho in the NAc Upregulated the Expressions of Accumbal GluN2B in Both Normal and CSDS Susceptible Mice}

To further confirm the action target of klotho on depressive-like behaviors, we explored the influences of elevating accumbal klotho on GluN2B expression in NAc of mice. We first detected the total and surface expressions of GluN2B in NAc in mice with klotho overexpression. As shown in Figure S4a, genetic overexpression of klotho in the NAc in normal mice did not affect the total expressions of GluN1 and GluN2A subunit, but significantly increased the levels of total GluN2B and PSD-95 ( $p<0.01$ vs. control group). Protein cross-linking assay revealed that the surface expression of GluN2B in NAc was significantly increased by klotho expression (Figure $S 4 b, p<0.01$ vs. susceptible group).

We next investigated whether elevation of klotho could restore total and surface expressions of GluN2B in NAc of susceptible mice. As shown in Figure 4a, genetic overexpression of klotho in the NAc in susceptible mice had no effect on the expression levels of GluN1 and GluN2A subunit, but significantly increased the total expressions of GluN2B and PSD-95 to levels comparable to those in control group ( $p<$ 0.01 vs. susceptible group). Results from the $\mathrm{BS}^{3}$ cross-linking experiments showed that genetic overexpression of klotho in the NAc in susceptible mice had no effect on the levels of GluN1 and GluN2A subunit in both the surface and intracellular pool (Figure $4 \mathrm{~b}$ and $\mathrm{c}$ ), but significantly increased the surface expression of GluN2B in NAc (Figure 4d, $p<0.01$ vs. susceptible group). Together with the above data, 
these results demonstrated that klotho in the NAc could modulate depressive-like behaviors by regulating the stability of surface GluN2B.

\section{Genetic Overexpression of Accumbal Klotho Reversed Altered Synaptic and Structural Plasticity in CSDS Susceptible Mice}

Previous studies have proved that synaptic molecular adaptations occurring in the neurons of NAC underlie susceptible and resilient responses to chronic stress ${ }^{8}$. We next performed a set of electrophysiological experiments to investigate the modulatory effects of klotho on synaptic plasticity at cortico-accumbal glutamatergic synapses. We firstly conducted whole-cell voltage-clamp recordings of synaptically evoked NMDAR-mediated excitatory postsynaptic currents (EPSCs) in NAc slices to examine the modulation of GluN2B-NMDARs. The relative contribution of GluN2B to EPSCs was determined by measuring the sensitivity of EPSCs to Ro 25-6981, a second-generation NMDAR blocker that displays a 3000 -fold higher specificity to the GluN2B subunit than to other subunits. Compared to control group, Ro 25-6981-sensitive EPSCs were substantially reduced in the NAc neurons of susceptible mice (control: 34.5 $\pm 5.9 \%$ of baseline, susceptible: $5.8 \pm 3.1 \%$ of baseline; $p<0.01$ ), and overexpression of klotho in the NAc significantly increased Ro 25-6981-sensitive EPSCs in susceptible mice to a level comparable to control ( $31.7 \pm 5.9 \%$ of baseline, $p<0.01 \mathrm{vs}$. susceptible group) (Figure $5 \mathrm{a}$ and $\mathrm{b}$ ). These results indicate that elevation of klotho could reverse the detrimental effect of CSDS on GluN2B-mediated function.

The PPF, a sensitive measure of the probability of transmitter release, is a common form of short-term presynaptic plasticity. As shown in Figure S5a and b, CSDS did not affect the PPF at cortico-accumbal pathway, and klotho overexpression in susceptible mice also had no effect on PPF, suggesting the lack of gross change in presynaptic function. Then the input-output relationships for field excitatory postsynaptic potentials amplitude, an indicator of synaptic efficacy, were compared among groups. We found that there was a slight decrease in the amplitude of field excitatory postsynaptic potentials in the corticoaccumbal pathway in susceptible mice, while genetic overexpression of accumbal klotho reversed the decreased basal synapse transmission in these mice (Figure S5c and d, $p<0.05$ vs. susceptible group).

Persistent impairment in NMDAR-dependent LTD in NAc was associated with behavioral adaptations to chronic stress ${ }^{8}$. Thus, the NMDAR-LTD in the NAc was compared in the control, susceptible, and KL-OE susceptible groups. Consistent with previous study, the NMDAR-LTD was disrupted in susceptible mice (Figure $5 \mathrm{~d}$ and $\mathrm{g}, p<0.05 \mathrm{vs}$. control group). Genetic overexpression of accumbal klotho significantly reversed the disrupted NMDAR-LTD in NAc of susceptible mice (Figure $5 f$ and $g, p<0.05$ vs. susceptible and GFP group). This result indicated that regulation of accumbal klotho could normalize the impaired synaptic plasticity that was associated with depressive behaviors.

To further characterize the mechanisms underlying the modulation of klotho in depressive-like behaviors, we investigated the effects of klotho elevation on structural plasticity in susceptible mice. Golgi staining was employed to determine dendrite spine density in neurons of NAc. Consistent with previous reported ${ }^{28}$, 
the dendrite spine density in neurons of NAc was significantly increased in susceptible mice (Figure $5 j$ and $k, p<0.05 \mathrm{vs}$. control group). Genetic overexpression of accumbal klotho in susceptible mice significantly reversed the alteration of dendrite spine density in NAc (Figure $5 \mathrm{j}$ and $\mathrm{k}, p<0.05 \mathrm{vs}$. susceptible mice), demonstrating that elevation of accumbal klotho normalized structural plasticity in susceptible mice.

\section{Blockade of GluN2B Abolished the Beneficial Effects of Klotho Elevation in CSDS Susceptible Mice}

We then investigated whether blocking GluN2B-containing NMDARs would eliminate the beneficial effects of klotho elevation in susceptible mice. Ro 25-6981 is usually used in the 0.1-1 $\mu \mathrm{M}$ range in brain in vitro ${ }^{29}$. Given that blockade of GluN2B may have impacts on synaptic plasticity and depressive-like behaviors, low dose of Ro 25-6981 was used in this study to avoid this possibility as far as possible. We showed that bilaterally intra-NAc infusion of low dose of Ro 25-6981 $(0.1 \mu \mathrm{M}, 0.5 \mu \mathrm{l})$ at $20 \mathrm{~min}$ before social interaction or sucrose preference test had no significant effect on the exploration time in interaction zone and the interaction ratio, as well as sucrose preference in susceptible mice, while it significantly abolished the increased social interaction and sucrose preference induced by klotho overexpression in these mice (Figure $6 \mathrm{a}$ and $\mathrm{b}, p<0.01 \mathrm{vs}$. KL-OE susceptible mice). Post-hoc comparisons using Bonferroni's test showed that both the interaction ratio and the sucrose consumption in klotho-overexpressed susceptible mice that were also treated with Ro 25-6981 were not different from those in GFP-treated susceptible mice $(p>0.05)$. In a separate set of experiments, we investigated the influence of an acute administration of Ro 25-6981 on NMDAR-dependent LTD in NAc of mice. Similar to the behavioral results, bath application of Ro 25-6981 (0.1 $\mu \mathrm{M})$ for 20 min did not affect LFS-induced LTD in slices from susceptible mice (Figure 6e), but significantly eliminated the benefit of klotho overexpression on LTD in these mice ( $p<0.01$ vs. KL-OE susceptible mice; Figure $6 \mathrm{~g}$ ). Post-hoc comparisons showed that the level of LTD in klotho-overexpressed susceptible mice with bath application of Ro 25-6981 was comparable to that of susceptible mice $(p>0.05)$. These data indicate that upregulation of GluN2B-NMDAR function mediated the beneficial effects of klotho elevation in susceptible mice.

\section{Discussion}

In the present study, we demonstrated a critical role of accumbal klotho in the pathogenesis of major depression. Exposure to chronic stress led to a significant downregulation of klotho in the NAc of mice and genetic knockdown of klotho in the NAc induced depressive-like behaviors. Overexpression of klotho in the NAc produced an antidepressive effect in normal mice, and ameliorated depressive-like performances and reversed the alterations of synaptic plasticity and structural morphology in CSDS susceptible mice. The molecular effects of klotho might be correlated with the regulation of GluN2Bcontaining NMDAR function because klotho knockdown in the NAc resulted in selective decreases of total and synaptic GluN2B expression, which were identical to those observed in susceptible mice, and elevation of accumbal klotho could reverse the changes of GluN2B expression. Moreover, a GluN2B- 
specific antagonist abolished the benefits of klotho elevation on depressive-like performances and accumbal LTD in susceptible mice. These findings demonstrate that klotho in the NAc modulates depressive-like behaviors by regulating the function of GluN2B-containing NMDARs.

Klotho is a single-pass membrane-bound protein that can be alternatively spliced to a membrane bound form ( $\mathrm{m}-\mathrm{KL}$ ) and secreted form $(\mathrm{s}-\mathrm{KL})^{30}$. It is released and cleaved in cerebrospinal fluid (CSF) and plasma, and has an influence on longevity and susceptibility to multiple complex disorders, including atherosclerosis, stroke and depression ${ }^{31}$. Klotho mutant mice display an increased level of oxidative stress in the hippocampus at 5 weeks of age and impaired cognitive function at 7 weeks ${ }^{16}$. Women under high chronic stress had significantly lower levels of serum klotho when compared to low-stress controls $^{20}$. The levels of klotho in the CSF were also significantly increased in geriatric patients with severe depression after electroconvulsive therapy, a highly effective antidepressant treatment strategy 22 . In this study, we found that chronic stress resulted in a significant decrease in klotho expression in the NAc. Previous studies have demonstrated that blunted responses in the NAc to gain were observed in depressed individuals ${ }^{32}$ as well as the offspring and first-degree relatives of depressed individuals ${ }^{33}$. Rappaport et al. reported that current depression severity was associated with hyporeactivity in the NAc in response to the anticipation of a reward ${ }^{34}$. We showed that genetic knockdown of klotho in the NAc induced depressive-like behaviors in mice, and genetic overexpression of accumbal klotho obviously ameliorated depressive-like behaviors in susceptible mice. These data indicate that reduced nucleus accumbens klotho contributes to the pathogenesis of major depression.

Synaptic plasticity is the activity-dependent modification of the strength or efficacy of synaptic transmission at synapses and has been demonstrated to play a central role in the capacity of the brain to incorporate transient experiences into persistent memory traces ${ }^{35}$. It has been proposed that activitydependent remodeling of excitatory synapses and associated dendritic spines is impaired during chronic stress, leading to neurological circuit disorders in the brain and the onset of depression symptoms ${ }^{36}$. NAC neurons receive glutamatergic inputs arising from limbic and cortical regions. Stress-induced dysfunction in the synaptic plasticity of cortico-accumbal glutamatergic synapse is implicated in the symptomology of depression ${ }^{37}$. Consistent with previous report ${ }^{8}$, we showed that there was a significant decrease in excitatory postsynaptic responses and an impairment of NMDAR-dependent LTD in the NAc of susceptible mice. Overexpression of klotho in the NAc significantly reversed the reduced postsynaptic responses and impaired LTD in susceptible mice, indicating that klotho elevation could restore the synaptic function in the NAc of susceptible mice. Alterations in synaptic strength or connectivity of neurons are responsible for the long-lasting behavioral symptoms induced by chronic stress ${ }^{28,38-40}$. After chronic social defeat stress, medium spiny neurons (MSNs) of NAc exhibit increased spine density that is correlated with enhanced depressive behaviors ${ }^{40,41}$. We found that the dendrite spine density in the MSNs of NAc was significantly increased in susceptible mice, while genetic overexpression of accumbal klotho substantially reversed the alteration of dendrite spine density in these mice, demonstrating elevation of accumbal klotho could normalize the structural plasticity in depressed mice. These results 
that klotho elevation reversed the disruptions of synaptic and structural plasticity in susceptible mice provided supporting evidence for the benefit of elevating klotho on depressive-like behaviors.

Previous data from our study showed that the reduction of GluN2B in the NAc can aggravate depressivelike behavior in mice. It is worth noting that systemic administration of NMDA receptor antagonist, such as Ro 25-6981 and ketamine, is capable of exerting significant antidepressant effects in both model animals and depressive patients ${ }^{42}$. This fact seems to conflict with our finding. However, there are some possible reasons to explain this discrepancy. Firstly, the mechanisms by which ketamine exert antidepressant effects are complex. Recent studies suggest that the effects of ketamine on depression cover not only NMDAR antagonism, but also include glutamate surge, reduced inhibitory GABAergic transmission, AMPAR-mediated increase in mTOR-dependent neuroplasticity as well as BDNF release ${ }^{43-}$ 47. On the other hand, different brain regions might exert different effects due to different structural components. A major proportion of NAc neurons are $\mathrm{MSNs}^{48}$. During the course of depression, NAc region would undergo some changes in protein expressions and functional connectivity that are different from other brain regions. For example, chronic stress causes a reduction in hippocampal BDNF expression and spine density 49,50 , while increased BDNF expression and dendrite spine density were observed in the NAc after chronic stress ${ }^{51}$. Our previous study has also demonstrated that chronic stress resulted in a long-lasting reduction of GluN2B in the NAc, which could be restored by fluoxetine treatment, and unsusceptible mice showed patterns of GluN2B regulation that overlapped dramatically with those seen with fluoxetine treatment ${ }^{8}$. In this study, we showed that chronic stress caused a parallel change in GluN2B and klotho expression in the NAc and the total and surface expressions of GluN2B in the NAc can be regulated by altering klotho levels via AAV-mediated knockdown or overexpression. Furthermore, treatment with low dose of Ro 25-6981 had no significant effect on the depressive-like behaviors and NMDAR-LTD in susceptible mice, but significantly abolished the beneficial effects of klotho overexpression in these mice, indicating that GluN2B was the action target of klotho in modulation of depressive-like behaviors. However, how klotho regulates the levels of total GluN2B protein and enriches GluN2B within synapses, directly or indirectly, remains to be determined but may implicate regulations of translation, posttranslational modification, recycling, or trafficking of the subunit. It also remains to be determined whether the effects of klotho elevation on GluN2B are mediated by the transmembrane or secreted form of klotho.

Taken together, we preliminarily demonstrated that klotho in the NAc modulate depressive-like behaviors by regulating the function of GluN2B-containing NMDARs. This finding provides novel insights into the pathogenesis of major depression and regulation of klotho in the NAc might be a strategy for the treatment of depression. However, how chronic stress causes a change in the expression of klotho in the NAc still needs further study.

\section{Declarations}

\section{Data availability}


The authors confirm that the data supporting the findings of this study are available within the article and the Supplementary Material. Additional data related to this paper may be requested from the authors.

\section{Acknowledgements}

This study was supported by grants from the National Natural Science Foundation of China (82060258, 81760254,81760256 and 81960256). It was also supported by the Jiangxi Provincial Natural Science Foundation (20202BAB206026, 20202BAB216012 and 20202BBG73022). The study also received support by grants from the Jiangxi Provincial Clinical Research Center (2020BCG74002) and the Academic and Technical Leaders of Major Disciplines Foundation (20204BCJL22049).

\section{Author contributions}

Y.-J.Y., B.W. and W.W. designed the research; H.-J.W., L.-E.L., W.-N.W., J.-Q.Z., C.-N.C., Y.-H.L., S.-Z.J., J.-W.X. and Z.-M.Y. performed the research; H.-J.W., L.-E.L., and Y.-J.Y. analyzed data; and H.-J.W. and Y.-J.Y. wrote the paper.

\section{Conflict of interest}

There is no conflict of interest.

\section{References}

1. Slavich GM, Irwin MR. From stress to inflammation and major depressive disorder: a social signal transduction theory of depression. Psychol Bull 2014; 140(3): 774-815.

2. Murray CJ, Lopez AD. Alternative projections of mortality and disability by cause 1990-2020: Global Burden of Disease Study. Lancet 1997; 349(9064): 1498-1504.

3. Otte C, Gold SM, Penninx BW, Pariante CM, Etkin A, Fava M et al. Major depressive disorder. Nat Rev Dis Primers 2016; 2: 16065.

4. Zhou Y, Zhu H, Liu Z, Chen X, Su X, Ma C et al. A ventral CA1 to nucleus accumbens core engram circuit mediates conditioned place preference for cocaine. Nat Neurosci 2019; 22(12): 1986-1999.

5. Steephen JE, Manchanda R. Differences in biophysical properties of nucleus accumbens medium spiny neurons emerging from inactivation of inward rectifying potassium currents. $J$ Comput Neurosci 2009; 27(3): 453-470.

6. Cho S, Hachmann JT, Balzekas I, In MH, Andres-Beck LG, Lee KH et al. Resting-state functional connectivity modulates the BOLD activation induced by nucleus accumbens stimulation in the swine brain. Brain Behav 2019; 9(12): e01431.

7. Krishnan V, Han MH, Graham DL, Berton O, Renthal W, Russo SJ et al. Molecular adaptations underlying susceptibility and resistance to social defeat in brain reward regions. Cel/2007; 131(2): 391-404. 
8. Jiang B, Wang W, Wang F, Hu ZL, Xiao JL, Yang S et al. The stability of NR2B in the nucleus accumbens controls behavioral and synaptic adaptations to chronic stress. Biol Psychiatry 2013; 74(2): 145-155.

9. Bagot RC, Parise EM, Pena CJ, Zhang HX, Maze I, Chaudhury D et al. Ventral hippocampal afferents to the nucleus accumbens regulate susceptibility to depression. Nat Commun 2015; 6: 7062.

10. Muir J, Tse YC, lyer ES, Biris J, Cvetkovska V, Lopez J et al. Ventral Hippocampal Afferents to Nucleus Accumbens Encode Both Latent Vulnerability and Stress-Induced Susceptibility. Biol Psychiatry 2020; 88(11): 843-854.

11. van Beugen BJ, Qiao X, Simmons DH, De Zeeuw Cl, Hansel C. Enhanced AMPA receptor function promotes cerebellar long-term depression rather than potentiation. Learn Mem 2014; 21(12): 662667.

12. Siddoway $B$, Hou H, Xia H. Molecular mechanisms of homeostatic synaptic downscaling. Neuropharmacology 2014; 78: 38-44.

13. Kuro-o M, Matsumura $Y$, Aizawa $H$, Kawaguchi $H$, Suga $T$, Utsugi T et al. Mutation of the mouse klotho gene leads to a syndrome resembling ageing. Nature 1997; 390(6655): 45-51.

14. Masuda $H$, Chikuda $H$, Suga $T$, Kawaguchi $H$, Kuro-o M. Regulation of multiple ageing-like phenotypes by inducible klotho gene expression in klotho mutant mice. Mech Ageing Dev 2005; 126(12): 1274-1283.

15. Torbus-Paluszczak M, Bartman W, Adamczyk-Sowa M. Klotho protein in neurodegenerative disorders. Neurol Sci 2018; 39(10): 1677-1682.

16. Nagai T, Yamada K, Kim HC, Kim YS, Noda Y, Imura A et al. Cognition impairment in the genetic model of aging klotho gene mutant mice: a role of oxidative stress. FASEB J 2003; 17(1): 50-52.

17. Li D, Jing D, Liu Z, Chen Y, Huang F, Behnisch T. Enhanced Expression of Secreted alpha-Klotho in the Hippocampus Alters Nesting Behavior and Memory Formation in Mice. Front Cell Neurosci 2019; 13: 133.

18. Dubal DB, Yokoyama JS, Zhu L, Broestl L, Worden K, Wang D et al. Life extension factor klotho enhances cognition. Cell Rep 2014; 7(4): 1065-1076.

19. Dubal DB, Zhu L, Sanchez PE, Worden K, Broestl L, Johnson E et al. Life extension factor klotho prevents mortality and enhances cognition in hAPP transgenic mice. J Neurosci 2015; 35(6): 23582371.

20. Prather AA, Epel ES, Arenander J, Broestl L, Garay BI, Wang D et al. Longevity factor klotho and chronic psychological stress. Transl Psychiatry 2015; 5: e585.

21. Paroni G, Seripa D, Fontana A, D'Onofrio G, Gravina C, Urbano M et al. Klotho Gene and Selective Serotonin Reuptake Inhibitors: Response to Treatment in Late-Life Major Depressive Disorder. Mol Neurobiol 2017; 54(2): 1340-1351.

22. Hoyer C, Sartorius A, Aksay SS, Bumb JM, Janke C, Thiel M et al. Electroconvulsive therapy enhances the anti-ageing hormone Klotho in the cerebrospinal fluid of geriatric patients with major depression. Eur Neuropsychopharmacol 2018; 28(3): 428-435. 
23. Imura A, Iwano A, Tohyama O, Tsuji Y, Nozaki K, Hashimoto N et al. Secreted Klotho protein in sera and CSF: implication for post-translational cleavage in release of Klotho protein from cell membrane. FEBS Lett 2004; 565(1-3): 143-147.

24. Leon J, Moreno AJ, Garay BI, Chalkley RJ, Burlingame AL, Wang D et al. Peripheral Elevation of a Klotho Fragment Enhances Brain Function and Resilience in Young, Aging, and alpha-Synuclein Transgenic Mice. Cell Rep 2017; 20(6): 1360-1371.

25. El-Husseini AE, Schnell E, Chetkovich DM, Nicoll RA, Bredt DS. PSD-95 involvement in maturation of excitatory synapses. Science 2000; 290(5495): 1364-1368.

26. D'Mello R, Marchand F, Pezet S, McMahon SB, Dickenson AH. Perturbing PSD-95 interactions with NR2B-subtype receptors attenuates spinal nociceptive plasticity and neuropathic pain. Mol Ther 2011; 19(10): 1780-1792.

27. Hardingham GE, Fukunaga Y, Bading $H$. Extrasynaptic NMDARs oppose synaptic NMDARs by triggering CREB shut-off and cell death pathways. Nat Neurosci 2002; 5(5): 405-414.

28. Christoffel DJ, Golden SA, Dumitriu D, Robison AJ, Janssen WG, Ahn HF et al. IkappaB kinase regulates social defeat stress-induced synaptic and behavioral plasticity. J Neurosci 2011; 31(1): 314-321.

29. Volianskis A, Bannister N, Collett VJ, Irvine MW, Monaghan DT, Fitzjohn SM et al. Different NMDA receptor subtypes mediate induction of long-term potentiation and two forms of short-term potentiation at CA1 synapses in rat hippocampus in vitro. J Physio/ 2013; 591(4): 955-972.

30. Masso A, Sanchez A, Gimenez-Llort L, Lizcano JM, Canete M, Garcia B et al. Secreted and Transmembrane alphaKlotho Isoforms Have Different Spatio-Temporal Profiles in the Brain during Aging and Alzheimer's Disease Progression. PLoS One 2015; 10(11): e0143623.

31. Pavlatou MG, Remaley AT, Gold PW. Klotho: a humeral mediator in CSF and plasma that influences longevity and susceptibility to multiple complex disorders, including depression. Trans/ Psychiatry 2016; 6(8): e876.

32. Keren H, O'Callaghan G, Vidal-Ribas P, Buzzell GA, Brotman MA, Leibenluft E et al. Reward Processing in Depression: A Conceptual and Meta-Analytic Review Across fMRI and EEG Studies. Am J Psychiatry 2018; 175(11): 1111-1120.

33. Luking KR, Pagliaccio D, Luby JL, Barch DM. Depression Risk Predicts Blunted Neural Responses to Gains and Enhanced Responses to Losses in Healthy Children. J Am Acad Child Adolesc Psychiatry 2016; 55(4): 328-337.

34. Rappaport BI, Kandala S, Luby JL, Barch DM. Brain Reward System Dysfunction in Adolescence: Current, Cumulative, and Developmental Periods of Depression. Am J Psychiatry 2020; 177(8): 754763.

35. Citri A, Malenka RC. Synaptic plasticity: multiple forms, functions, and mechanisms. Neuropsychopharmacology 2008; 33(1): 18-41.

36. Vose LR, Stanton PK. Synaptic Plasticity, Metaplasticity and Depression. Curr Neuropharmacol 2017; 15(1): 71-86. 
37. Ma K, Zhang H, Wei G, Dong Z, Zhao H, Han X et al. Identification of key genes, pathways, and miRNA/mRNA regulatory networks of CUMS-induced depression in nucleus accumbens by integrated bioinformatics analysis. Neuropsychiatr Dis Treat 2019; 15: 685-700.

38. Holtmaat A, Svoboda K. Experience-dependent structural synaptic plasticity in the mammalian brain. Nat Rev Neurosci 2009; 10(9): 647-658.

39. Khibnik LA, Beaumont M, Doyle M, Heshmati M, Slesinger PA, Nestler EJ et al. Stress and Cocaine Trigger Divergent and Cell Type-Specific Regulation of Synaptic Transmission at Single Spines in Nucleus Accumbens. Biol Psychiatry 2016; 79(11): 898-905.

40. Qiao H, Li MX, Xu C, Chen HB, An SC, Ma XM. Dendritic Spines in Depression: What We Learned from Animal Models. Neural Plast 2016; 2016: 8056370.

41. Fox ME, Figueiredo A, Menken MS, Lobo MK. Dendritic spine density is increased on nucleus accumbens D2 neurons after chronic social defeat. Sci Rep 2020; 10(1): 12393.

42. Autry AE, Adachi M, Nosyreva E, Na ES, Los MF, Cheng PF et al. NMDA receptor blockade at rest triggers rapid behavioural antidepressant responses. Nature 2011; 475(7354): 91-95.

43. Abdallah CG, Sanacora G, Duman RS, Krystal JH. The neurobiology of depression, ketamine and rapid-acting antidepressants: Is it glutamate inhibition or activation? Pharmacol Ther 2018; 190: $148-158$.

44. Li N, Lee B, Liu RJ, Banasr M, Dwyer JM, Iwata M et al. mTOR-dependent synapse formation underlies the rapid antidepressant effects of NMDA antagonists. Science 2010; 329(5994): 959-964.

45. Cavalleri L, Merlo Pich E, Millan MJ, Chiamulera C, Kunath T, Spano PF et al. Ketamine enhances structural plasticity in mouse mesencephalic and human iPSC-derived dopaminergic neurons via AMPAR-driven BDNF and mTOR signaling. Mol Psychiatry 2018; 23(4): 812-823.

46. Zanos P, Gould TD. Mechanisms of ketamine action as an antidepressant. Mol Psychiatry 2018; 23(4): 801-811.

47. Gerhard DM, Pothula S, Liu RJ, Wu M, Li XY, Girgenti MJ et al. GABA interneurons are the cellular trigger for ketamine's rapid antidepressant actions. J Clin Invest 2020; 130(3): 1336-1349.

48. Soares-Cunha C, de Vasconcelos NAP, Coimbra B, Domingues AV, Silva JM, Loureiro-Campos E et al. Nucleus accumbens medium spiny neurons subtypes signal both reward and aversion. $\mathrm{Mol}$ Psychiatry 2020; 25(12): 3241-3255.

49. Hamani C, Machado DC, Hipolide DC, Dubiela FP, Suchecki D, Macedo CE et al. Deep brain stimulation reverses anhedonic-like behavior in a chronic model of depression: role of serotonin and brain derived neurotrophic factor. Biol Psychiatry 2012; 71(1): 30-35.

50. De Miguel Z, Haditsch U, Palmer TD, Azpiroz A, Sapolsky RM. Adult-generated neurons born during chronic social stress are uniquely adapted to respond to subsequent chronic social stress. $\mathrm{Mol}$ Psychiatry 2019; 24(8): 1178-1188.

51. Walsh JJ, Friedman AK, Sun H, Heller EA, Ku SM, Juarez B et al. Stress and CRF gate neural activation of BDNF in the mesolimbic reward pathway. Nat Neurosci 2014; 17(1): 27-29. 
Figures

2

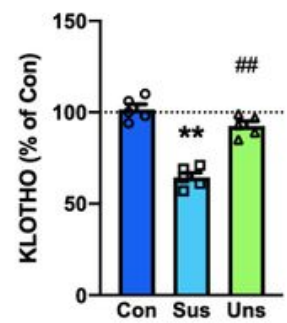

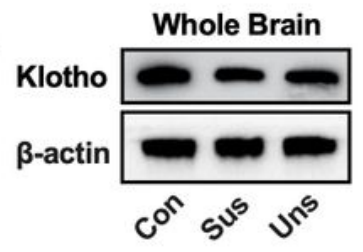

b
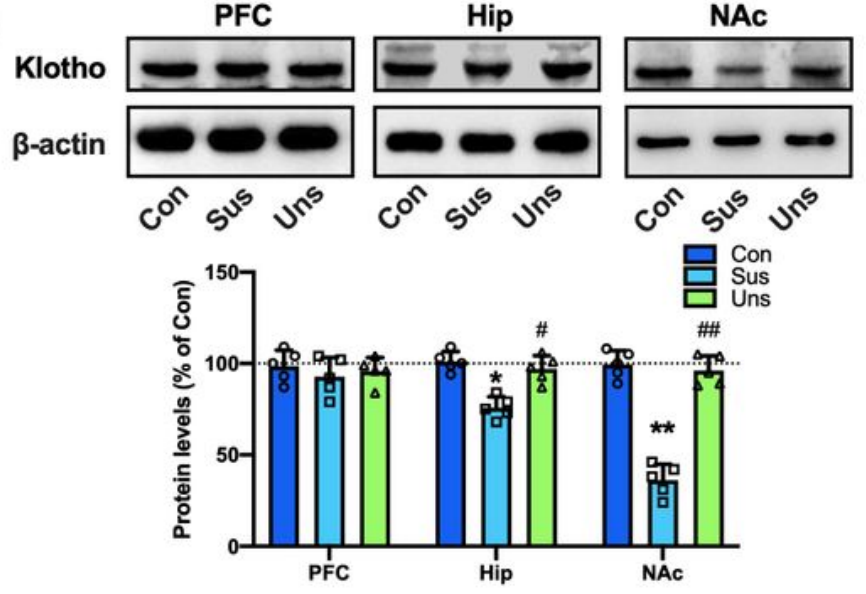

C

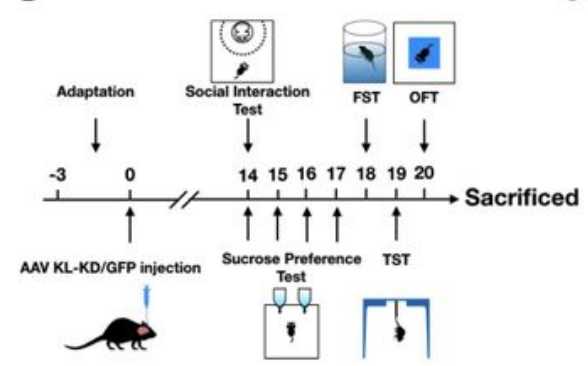

d
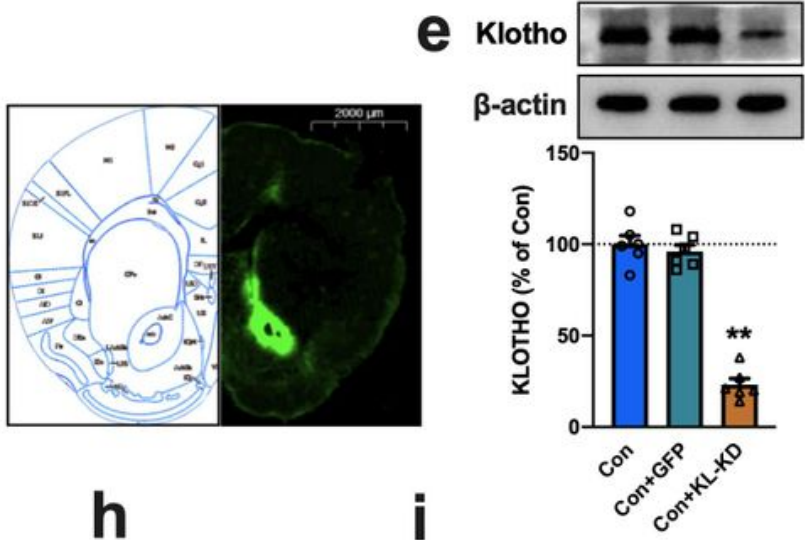

f

$9 \quad$ FST $\quad h$
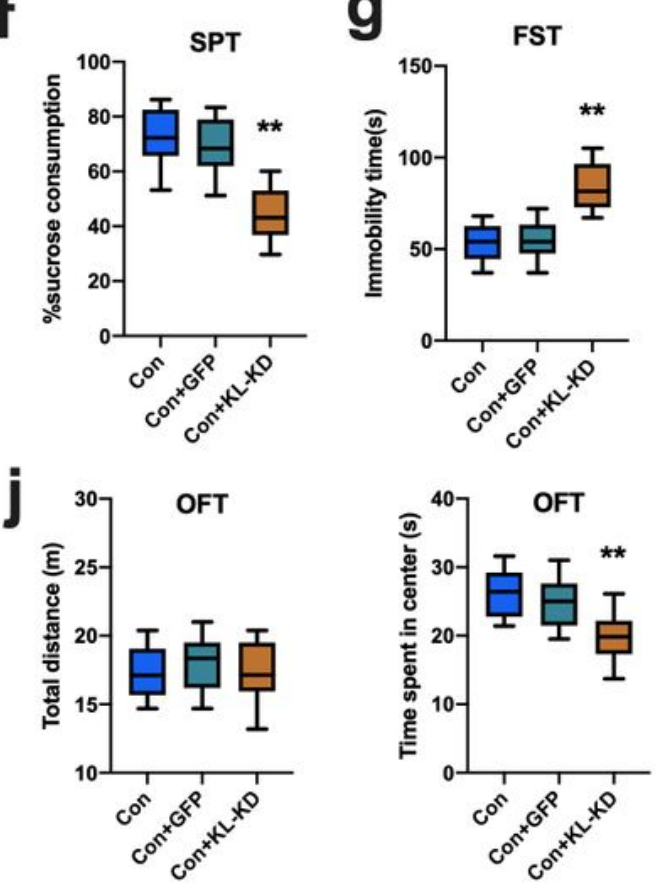
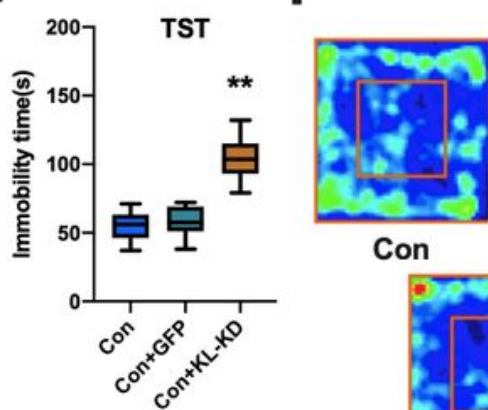

Con

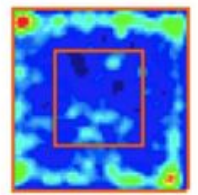

Con+KL-KD

\section{Figure 1}

Downregulation of klotho in the NAc contributes to the depressive-like behaviors in mice. (a)

Representative images of western blotting and histograms showing the levels of klotho protein in whole brains from the control, susceptible and unsusceptible mice ( $n=5$ mice per group). (b) The expressions 
of klotho protein in the PFC, Hip, and NAc from the control, susceptible and unsusceptible mice $(\mathrm{n}=5$ mice per group). (c) Timeline of the experimental procedures. (d) Fluorescence image of a fixed brain section which expressed AAV-KL-KD in the NAc 14 days after stereotactic injection (Scale bar $=2000 \mu \mathrm{m}$ ). (e) Representative western blotting images and histograms showing the protein expression of klotho in the NAc in each group ( $n=6$ mice per group). (f) KL-KD in the NAc significantly decreased sucrose preference in mice. $(g, h)$ KL-KD in the NAc increased the immobility time in FST $(g)$ and TST $(h)$ in mice. (i) Representative images of movement tracks in the OFT. (j) KL-KD in the NAc did not affect total travelled distance (left), but significantly decreased the time spent in the central area (right) in the OFT in mice. For $a, b$ and $e$, data are presented as normalized mean $\pm S E M$. For $f-j$, all box and whisker plot displays the median, first and third quartiles (boxes), and the min-max (whiskers). ${ }^{*} p<0.05$ and ${ }^{* *} p<0.01$ vs. control. ${ }^{\#} p<0.05$ and ${ }^{\# \#} p<0.01$ vs. susceptible group or GFP group.
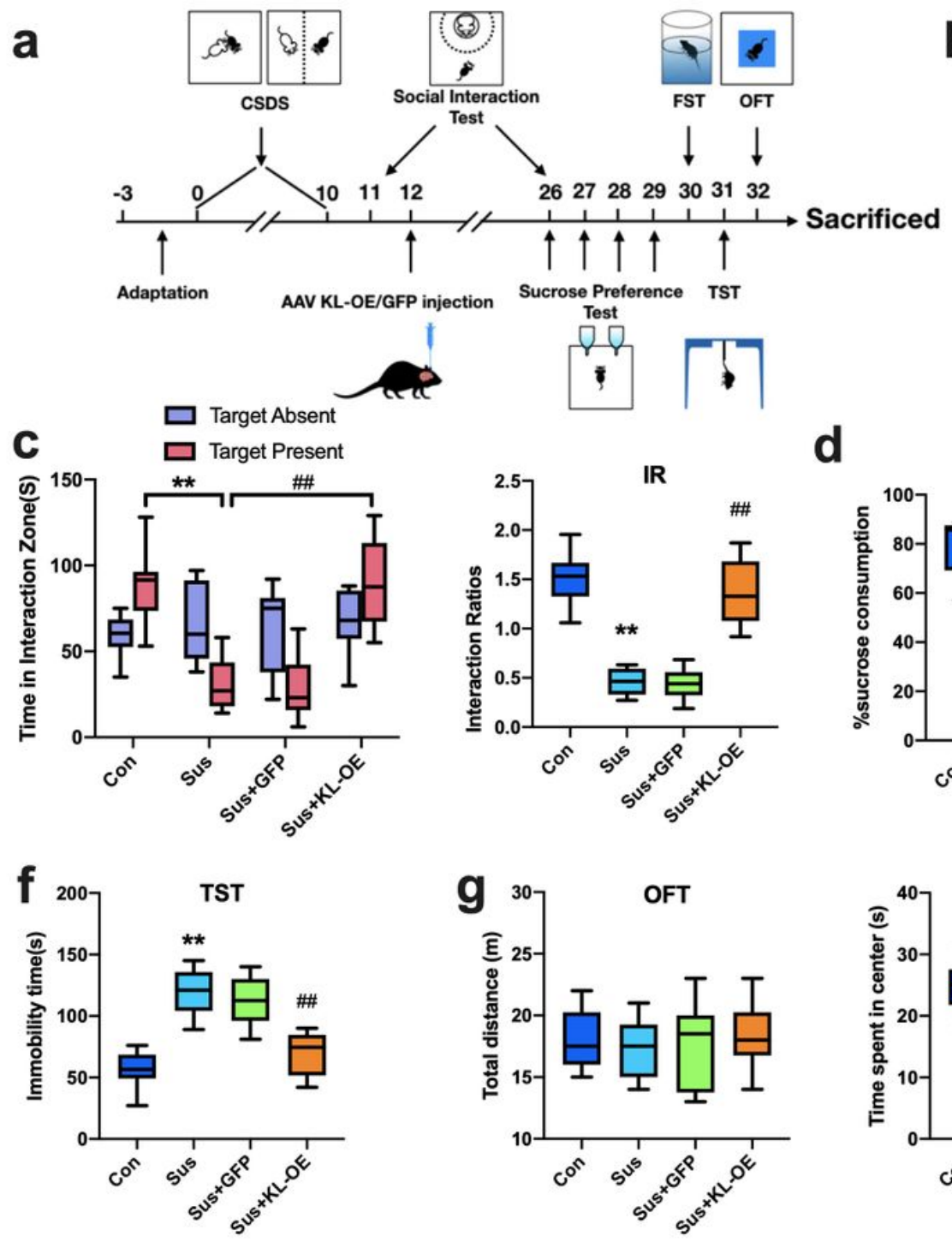

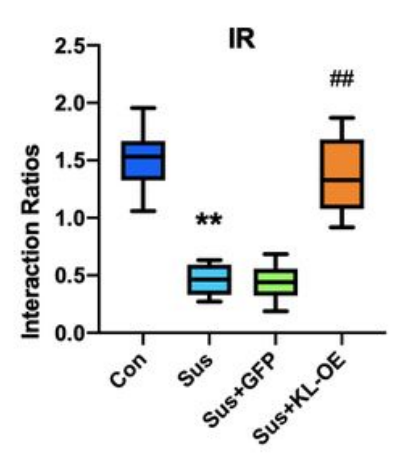

d
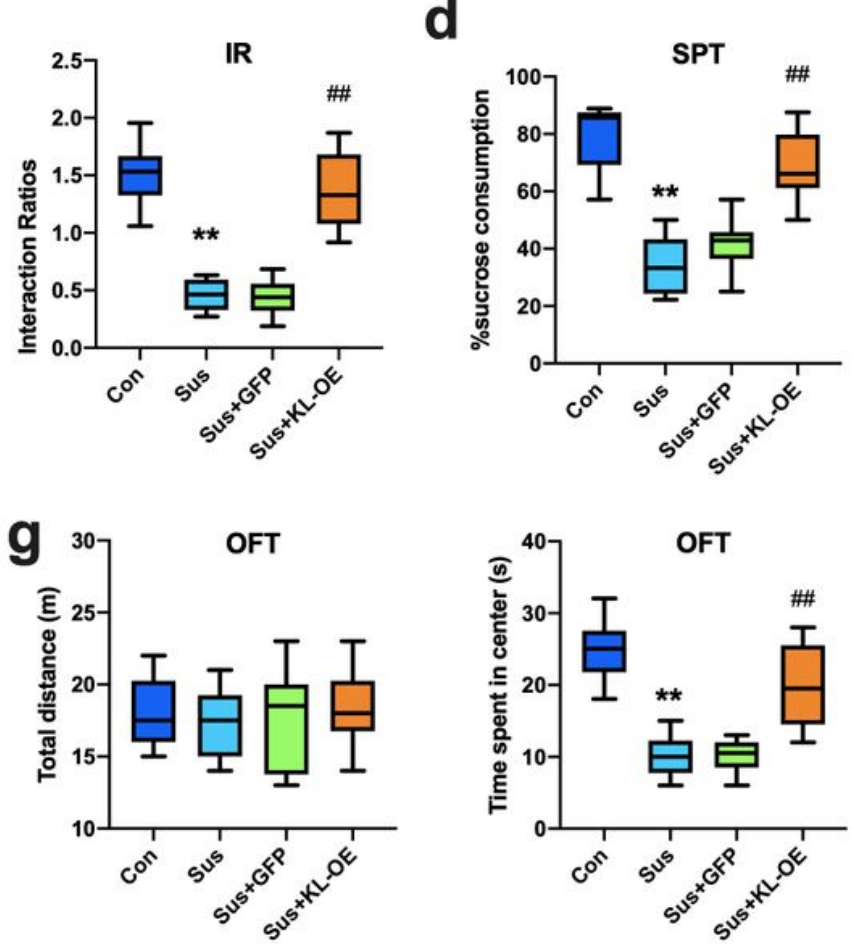
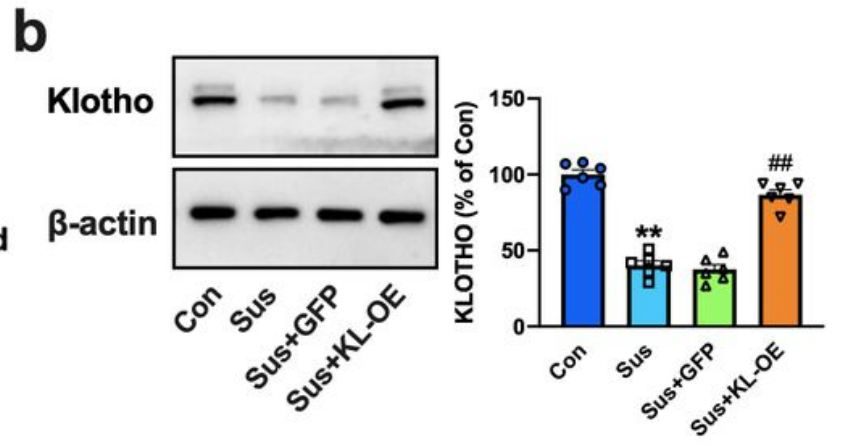

$\mathbf{e}$
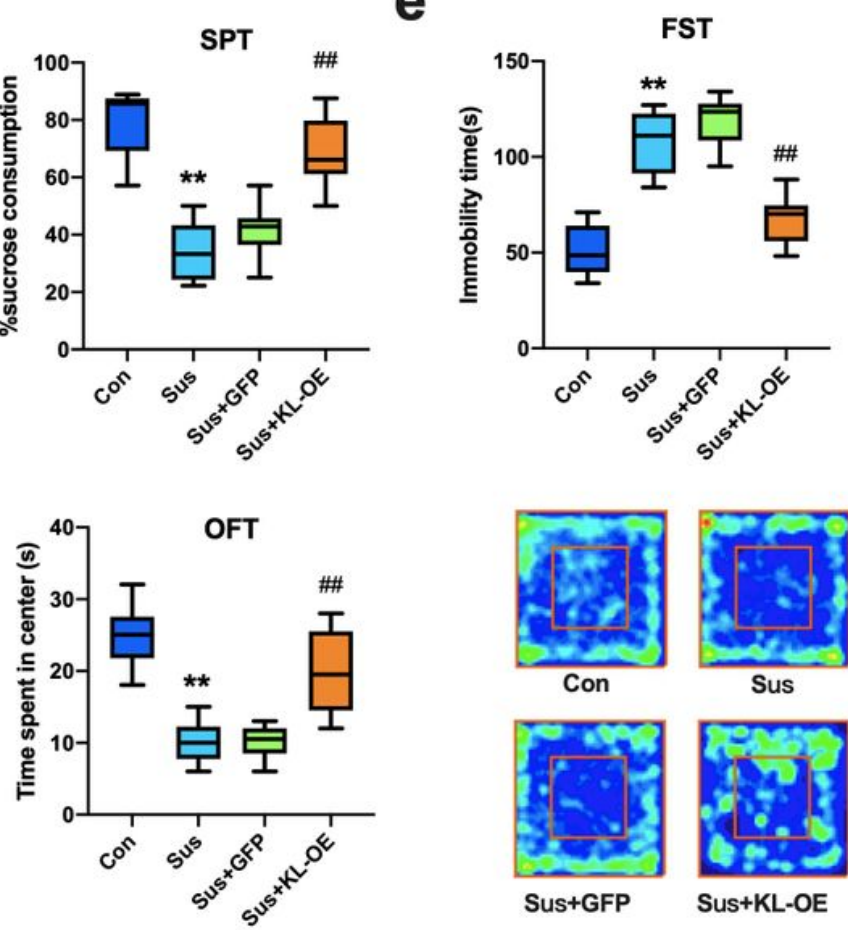

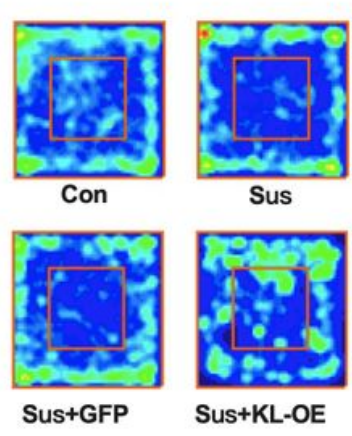

Figure 2

Genetic overexpression of klotho in the NAc ameliorates the depressive-like behaviors in CSDS susceptible mice. (a) Timeline of the experimental procedures. (b) Representative western blotting images 
and histograms showing the protein expression of klotho in the NAc in each group ( $\mathrm{n}=6$ mice per group). (c) KL-OE in the NAc significantly increased the time in interaction zone (left) and social interaction ratios (right) in CSDS susceptible mice. (d-f) KL-OE in the NAc significantly reversed the decreased sucrose preference (d) and the increased immobility time in FST (e) and TST (f) in CSDS susceptible mice. (g) KL$\mathrm{OE}$ in the NAc did not affect total travelled distance (left), but increased the time spent in the central area (middle) in OFT in CSDS susceptible mice. Representative images of movement tracks in OFT were shown in the right. For b, data were presented as normalized mean \pm SEM. For $c-g$, all box and whisker plot display the median, first and third quartiles (boxes), and the min-max (whiskers) ( $n=10$ mice per group). ${ }^{* *} p<0.01$ vs. control; ${ }^{\# \#} p<0.01$ vs. susceptible or susceptible-GFP group.
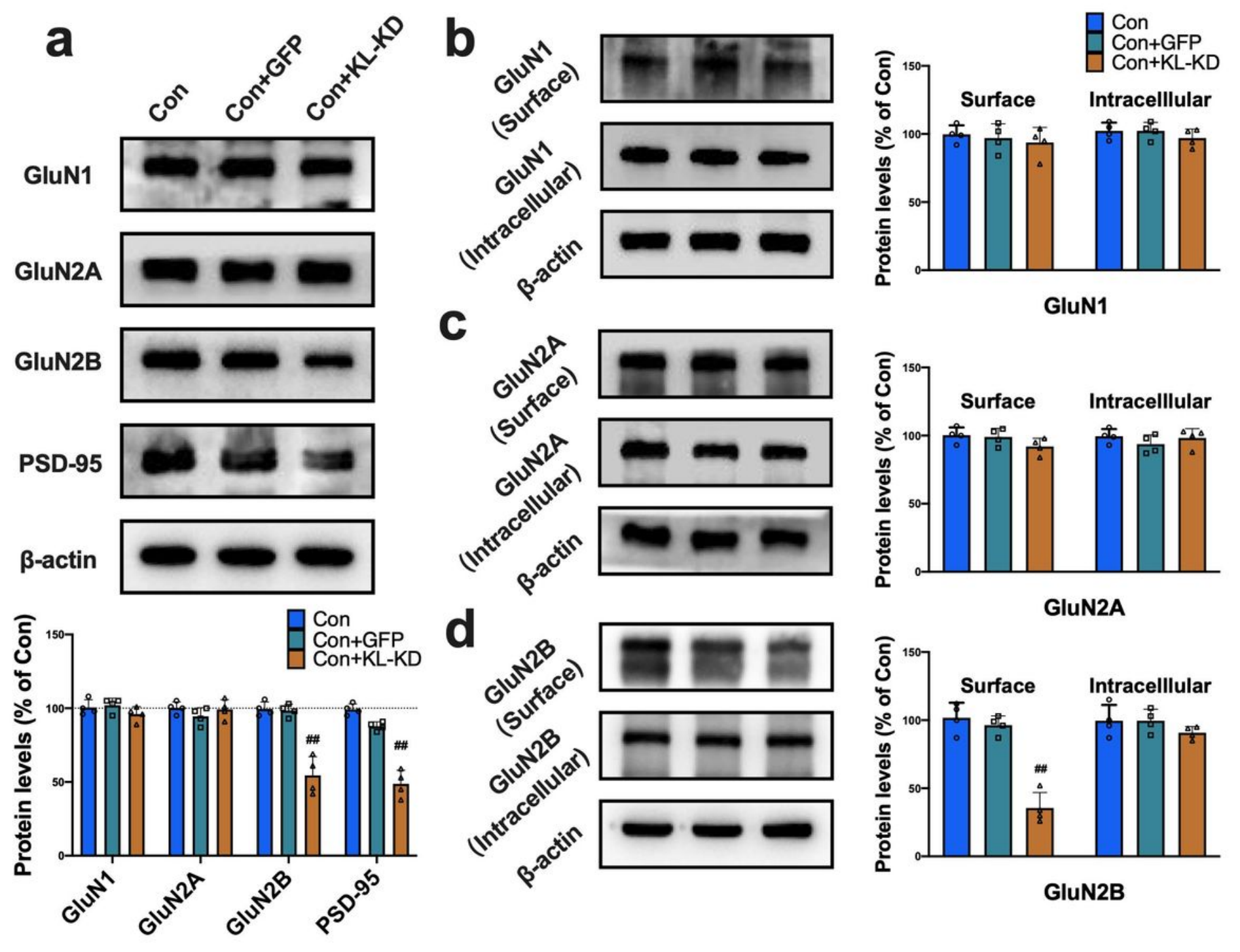

GluN2A

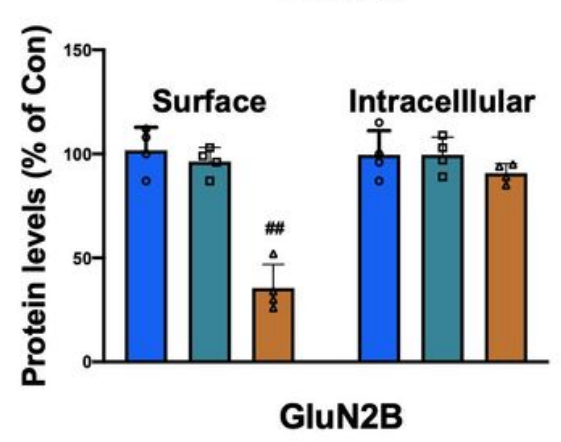

Figure 3

Genetic knockdown of klotho in the NAc selectively downregulated the expressions of total and surface GluN2B in normal mice. (a) Knockdown of accumbal klotho did not affect the total GluN1 and GluN2A protein levels, while it significantly decreased the total GluN2B and PSD-95 protein expression in the NAC ( $n=4$ mice per group). (b-c) Western blotting analysis revealed that genetic knockdown of accumbal 
klotho produced no effect on the expressions of GluN1 (b) and GluN2A (c) in both the surface and intracellular pools in NAc of mice. (d) Genetic knockdown of accumbal klotho significantly reduced GluN2B expression in the surface pool but not in the intracellular pool ( $n=4$ mice per group). All data were presented as normalized mean \pm SEM. ${ }^{\# \#} p<0.01$ vs. GFP group.
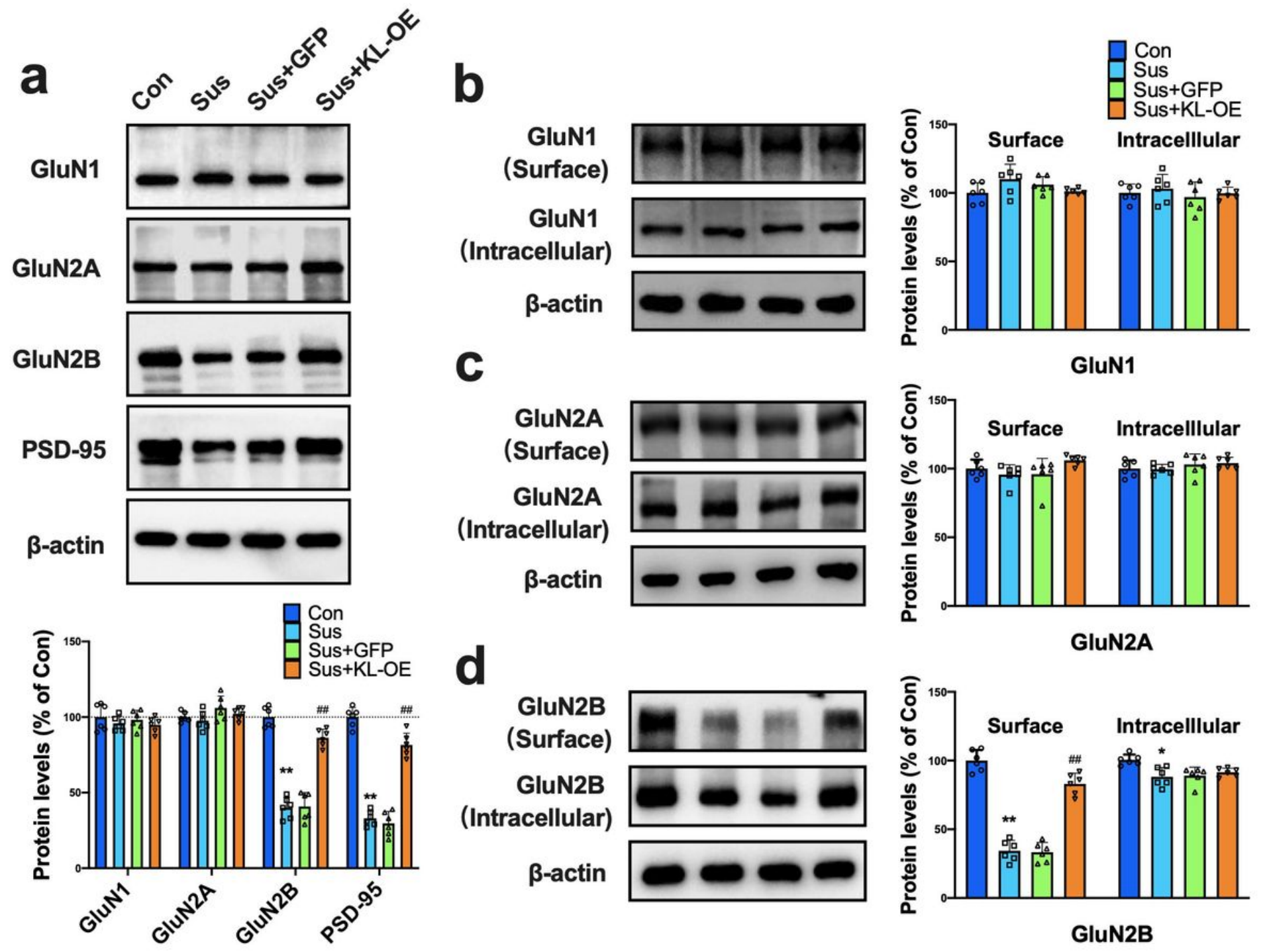

GluN2A

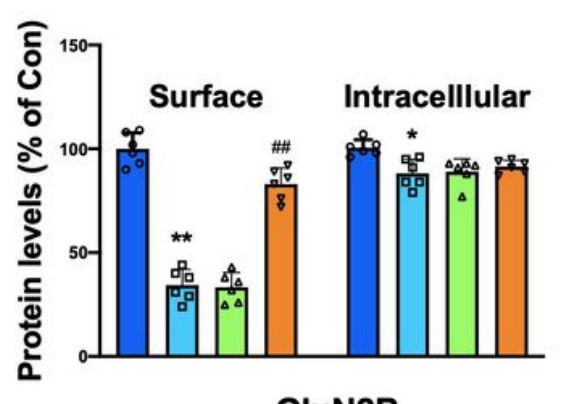

GluN2B

Figure 4

Genetic overexpression of klotho in the NAc reverses the reduction of GluN2B expression in CSDS susceptible mice. (a) Representative immunoblots showing the total protein expressions of GluN1, GluN2A, GluN2B and PSD-95 in the NAc of mice from each group. Statistical results show that CSDS decreased the protein expressions of GluN2B and PSD-95 in the NAc, while klotho overexpression reversed the effects of CSDS ( $n=6$ mice per group). (b-d) Representative immunoblots showing the expressions of GluN1 (b), GluN2A (c) and GluN2B (d) in both the surface and intracellular pool in the NAc. Statistical results show that CSDS caused a reduction of GluN2B in the surface pool of NAc, while klotho overexpression reversed the decline of surface GluN2B expression ( $n=6$ mice per group). All data were presented as normalized mean \pm SEM. ${ }^{* *} p<0.01$ vs. control; ${ }^{\# \#} p<0.01$ vs. susceptible group. 


\section{Figure 5}

Genetic overexpression of klotho in the NAc restores the CSDS-induced disruption of NMDAR-dependent LTD and dendritic spines density. ( $a, b)$ Representative traces (a) and quantitation (b) of isolated NMDAR EPSCs in the presence of NBQX $(10 \mu \mathrm{M})$ at baseline (black) and following perfusion with Ro 25-6981 (Ro $25 ; 0.1 \mu \mathrm{M})(\mathrm{red})$ in the same slices. Five slices from three mice in each group were analyzed. Compared to control group, CSDS susceptible mice displayed lower responses to stimulations and overexpression of accumbal klotho reversed the downregulation of input-output curves in these mice ( $n=5$ per group). (c- $f$ ) Averaged data show the LTD induced by LFS in control (c), Susceptible (d), Sus+GFP (e) and Sus+KL-OE group (f). The LFS consisting of a 1-Hz, 900-pulse trains at stimulus intensity. (g) The box and whiskers show the levels of normalized fEPSP slope 40 min after LFS from each group $(n=5$ per group). ( $h, i)$ Representative micro-photograph show stained dendrites in the NAc: (h) Scale bar $=500 \mu \mathrm{m}$, (i) Scale bar $=20 \mu \mathrm{m}$. (j) Representative dendritic spine images from control (j1), Sus (j2), Sus + GFP (j3) and Sus + $\mathrm{KL}-\mathrm{OE}$ group (j4). Scale bar $=5 \mu \mathrm{m}$. (k) Quantification of dendritic spine density in the NAc of mice from each group ( $\mathrm{n}=6$ mice per group). Each point was the normalized mean $\pm \mathrm{SEM}$. ${ }^{*} p<0.05$ and ${ }^{* *} p<0.01$ vs. control; $\#<0.05$ and ${ }^{\# \#} p<0.01$ vs. susceptible group.

\section{Figure 6}

Treatment with GluN2B antagonist abolishes the beneficial effects of klotho elevation on depressive-like behaviors and synaptic plasticity in CSDS susceptible mice. (a, b) Intra-NAc infusion of Ro 25-6981 (0.1 $\mu \mathrm{M}, 0.5 \mu \mathrm{l}) 20$ min before test did not affect the time in interaction zone and social interaction ratios (a) as well as sucrose preference (b) in CSDS susceptible mice, while it obviously abolished the enhancement effect of klotho overexpression on time in interaction zone and social interaction ratios (a) and sucrose preference (b) in these mice ( $\mathrm{n}=10$ mice per group). (c-g) NMDAR-dependent LTD recorded in the NAc in control (c), Sus + GFP (d), Sus + GFP + Ro-25 (e), Sus + KL-OE (f) and Sus + KL-OE + Ro-25 group (g). Bath application of Ro 25-6981 (0.1 $\mu \mathrm{M})$ for 20 min did not affect LFS-induced NMDARdependent LTD in slices from CSDS susceptible mice, but clearly abolished the beneficial effects of klotho overexpression on LTD in these mice. (h) The box and whiskers show the level of normalized fEPSP slope 40 min after LFS from each group ( $n=6$ per group). For a-d and $j$, all box and whisker plot display the median, first and third quartiles (boxes), and the min-max (whiskers), and other data were presented as normalized mean \pm SEM. ${ }^{\star *} p<0.01$ vs. control; ${ }^{\# \#} p<0.01$ vs. Sus + GFP group; ${ }^{\& \&} p<0.01$ vs. Sus + KL-OE group.

\section{Supplementary Files}

This is a list of supplementary files associated with this preprint. Click to download. 
- Supplementalinformation.docx 